\title{
TARSO-EPIPHYSIAL ACLASIS
}

\section{A Congenital Error of Epiphysial Development}

\author{
David Trevor, London, England
}

\author{
From the Orthopaedic Department, Charing Cross Hospital, \\ and the Institute of Orthopaedics, Roval National Orthopaedic Hospital
}

This condition is a congenital growth disorder of the tarsus and of the epiphyses. So far as can be ascertained from perusal of the literature it was first described by Mouchet and Belot in $\mathbf{1 9 2 6}$ in the case of a small boy, aged eighteen months, whose ankle was affected; they called it tarsomégalie. The disorder is more widespread than these authors appreciated and for that reason the term tarsomégalie, although descriptive enough of their own case, is not suitable when applied to the disorder as we now know it.

This contribution includes a clinical and radiological study with, in some cases, a report of the operative findings in eight patients suffering from tarso-epiphysial aclasis. Seven of the patients were boys and one was a girl; the youngest was first seen at the age of nine months and the oldest at ten years. The lower limbs alone were affected, the left five times and the right three times.

Distribution-The site generally involved was the knee or ankle joint-both knee and ankle together in five patients, the knee joint alone in two and the ankle joint alone in one. When there was change in both knee and ankle joints it was invariably found that the same side of the epiphyses was affected in both joints - the inner side of the knee with the inner side of the ankle, or the outer side of the knee with the outer side of the ankle. There was no instance of the inner side of the knee joint being involved with the outer side of the ankle, or vice versa. Of the seven patients with involvement of the knee joint, the medial condyle of the femur was affected in five (two with similar change in the medial half of the upper tibial epiphysis) and the lateral femoral condyle in two (one with similar change in the lateral half of the upper tibial epiphysis). Of the seven patients with involvement of the ankle joint the medial malleolus and medial half of the talus was affected in four (two with changes also in the tarsal navicular and cuneiform bones), and the lateral malleolus and lateral half of the talus in three. In one patient the medial half of the upper femoral epiphysis was affected. Clinical features-Medical advice was sought either by reason of deformity of the lower limb or of swelling in the region of the knee or ankle joints. There was no complaint of pain. The deformity depended upon the site of the lesion. Affection of the medial epiphyses of the knee joint caused genu valgum-one patient aged less than five years having four and a half inches of deformity, partly in the femur and partly in the tibia (Case 4). Affection of the lateral part of the lower femoral or upper tibial epiphyses caused genu varum. It was the bony mass from overgrowth of the epiphyses that accounted for deformity and also interfered with full movement of the joint (there being limitation of both flexion and extension movement in Case 4, of flexion movement alone in Case 5 and of extension movement alone in Case 8). Similarly, a bony mass on the medial side of the ankle joint caused severe valgus deformity with limitation of inversion movement, while involvement of the lateral aspect of the ankle joint caused varus deformity. The circumference of the limb was sometimes smaller than on the normal side, but there was no true shortening. Such investigations as the Wassermann reaction and the blood count showed no departure from the normal.

Radiographic appearances-These are both striking and characteristic. The changes are confined to the epiphyses; the metaphysis is normal. In the case of the knee joint there is 
enlargement of the femoral epiphysis, and sometimes of the tibial epiphysis (Cases 4,5 and 6 ). This enlargement grows from the epiphysis back towards the popliteal space above the epiphysial line, extending also inwards on the medial side and outwards on the lateral side. The appearances suggest a mass of cartilage tissue in which there are several foci of ossification. This is well illustrated in the youngest child (Case 8). In older children the separate foci have fused, thus giving rise to a more uniform appearance throughout the growth. The changes are seen in only half of the epiphysis: in two cases the medial half of the femoral epiphysis was affected anteriorly and the corresponding half of the tibial epiphysis was affected posteriorly. When the medial side of the ankle was affected the medial malleolus was enlarged, and on the medial side of the talus there was a surface growth extending medially, irregular in outline and similar to (but not so well defined as) the knee outgrowth. The whole talus was irregular and enlarged-sometimes twice the size of that on the opposite side and as big as the calcaneum. When the navicular and the cuneiforms were affected, singly or

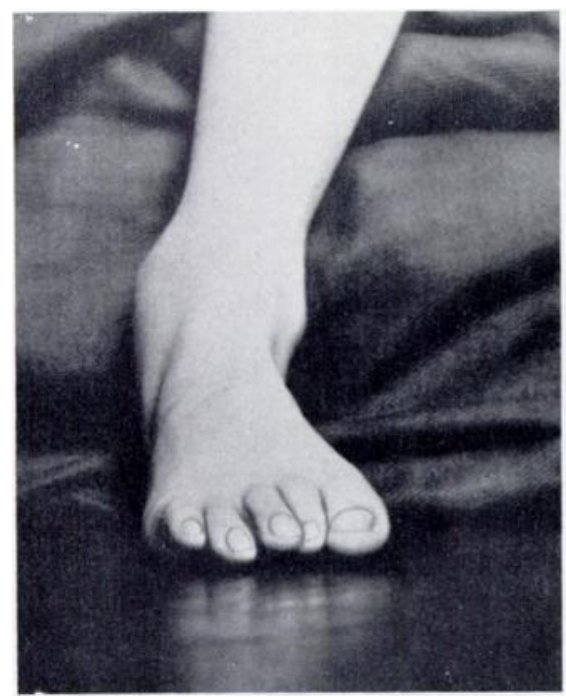

FIG. 1

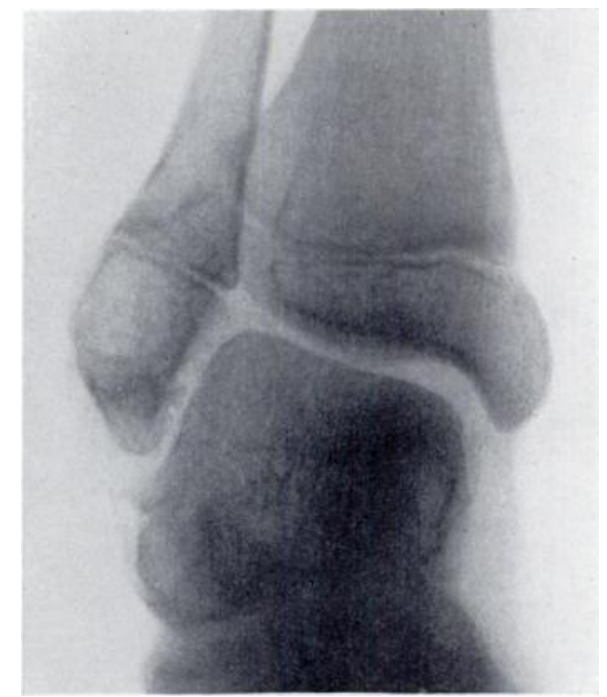

FIG. 2

Case 1. Figure 1-Right ankle showing enlargement of the lateral malleolus extending down to the talus; and Figure 2, enlargement of the medial malleolus and the lateral side of the talus with an irregular outgrowth from each.

together, the ossific nucleus appeared early; instead of one centre there were several small foci. When the lesion occurred in the lateral side of the ankle the radiographic changes were similar.

In Case 7 of this series the upper femoral epiphysis was irregularly enlarged, mainly on the medial side; this is especially interesting because the medial side of the lower femoral epiphysis, the medial side of the upper tibial epiphysis, the medial malleolus, the medial half of the talus, the navicular and cuneiforms were involved; this distribution may be of significance in considering the etiology of the condition.

The bony enlargements were observed macroscopically in the patients submitted to operation. In Case 1 the swelling on the outer side of the ankle consisted of a pedunculated "exostosis" about one inch in length, with smaller distinct nodules, some on the anterior margin of the lower end of the tibia; the part removed consisted largely of bone covered by cartilage, the surface of which was nodular. In Case 2 the swelling was smooth, rounded and attached to the medial femoral condyle by a wide, short pedicle. It consisted of cartilage with a few bony nuclei in its substance. In Case 4 the medial femoral condyle was enlarged, nodular and bluish in places; no distinction could be made between the swelling and the

VOL. 32 B, NO. 2, MAY 1950 
medial femoral condyle except at its lateral margin in the intercondylar notch; the appearances of the adjacent portion of the upper end of the tibia were similar. When later the ankle region was explored a grossly nodular bony swelling was removed from the lower end of the medial side of the tibia; the upper and medial sides of the talus were similarly nodulated. In Case 6 the lateral femoral condyle was enlarged; the epiphysial cartilage seemed normal except for many small nodules. In Case 8 (the youngest child in the series) the medial femoral condyle was enlarged but smooth.

In Cases $1,2,4$ and 6 a part of the bony enlargement was examined microscopically; all the sections showed normal hyaline cartilage with areas of ossification.

\section{CASE REPORTS}

Case 1. (Reported by the courtesy of Sir Thomas Fairbank. Figures 1 and 2 .) Boy aged ten years. Had noticed swelling of the right ankle for two years; the foot had begun to "turn over." On examination there was

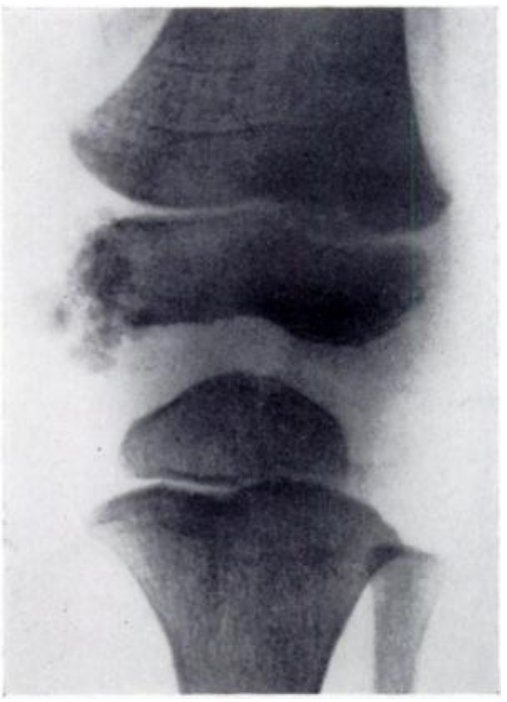

Fig. 3

Case 2. Ieft knee showing enlargement of the medial half of the lower femoral epiphysis. There are several foci of ossification. varus deformity of the right ankle, with a bony enlargement of the lateral malleolus and of the talus. The foot could not be everted. The right thigh and calf were smaller in circumference than the left. The only other abnormality found on general examination was a cataract of the right eve. Radiographs confirmed that the bony enlargement extended from the lateral malleolus and that the lateral sides of the talus and calcaneum were also involved. The enlargement was removed.

Case 2. (Reported by the courtesy of Sir Thomas Fairbank. Figure 3.) Boy aged four and a half years. For six weeks there had been a painless swelling on the inner side of the left knee; movement was full and painless. The swelling appeared to arise from the medial femoral condyle; this was confirmed by radiographic examination. The bony prominence was excised. Subsequent radiographs suggested some persistent bony nodules on the surface of the condyle, but a further exploration, more posteriorly, showed only a smooth cartilaginous convexity. When this patient was examined recently, many years after the operation, the function of the knee joint was normal.

Case 3. (Reported by the courtesy of Sir Reginald Watson-Jones. Figures 4, 5 and 6.) Boy aged eight years. Advice was sought for a swelling on the lateral side of the right knee. The boy was stated to have fallen on the knee from a chair at the age of fifteen months, and later to have injured the same knee in falling from his brother's back. On examination there was a bony swelling at the lateral side of the lateral femoral condyle. The movements of the knee joint were normal except for excessive rotation of the extended joint. There was a similar bony mass below and behind the lateral malleolus. The movements of the ankle joint were limited to 20 degrees but were painless. There was marked varus deformity of the foot, and bowing of the right tibia.

Case 4. (Reported by the courtesy of Miss M. Forrester-Brown. Figures 7 and 8.) Boy aged four and a half years when first seen. Left genu valgum noticed at the age of two years. The deformity had increased gradually. On examination there was enlargement of the medial femoral condyle and tibial tuberosity. There was marked valgus deformity, with four and a half inches separation between the malleoli. The knee joint was held flexed; 


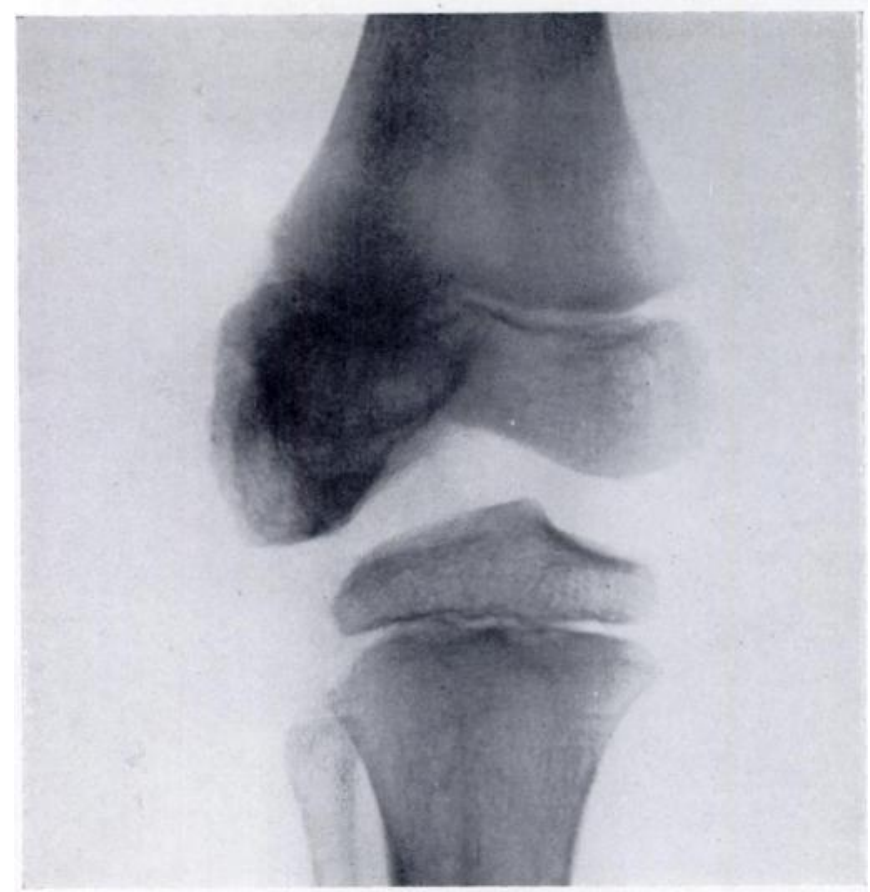

FIG. 4

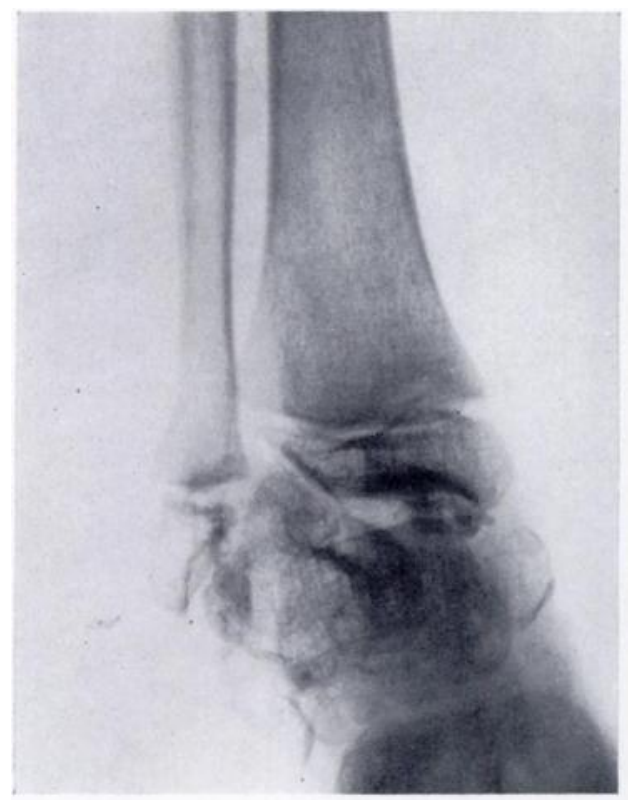

FIG. 5

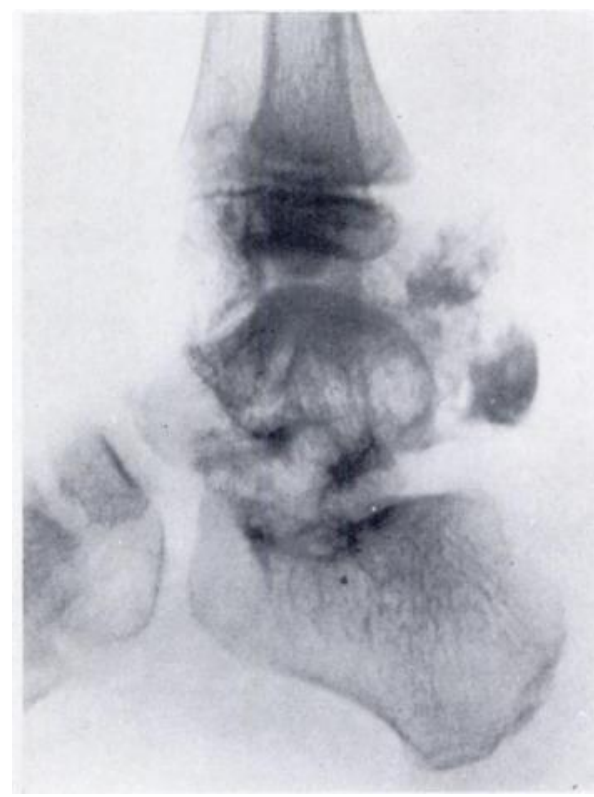

FIG. 6

Case 3. Figure 4-Knee showing an enlargement of the lateral femoral condvle. Figures 5 and 6 are anterior and lateral views showing a cartilaginous-bony mass extending from the lateral half of the talus below and behind the lateral malleolus. 


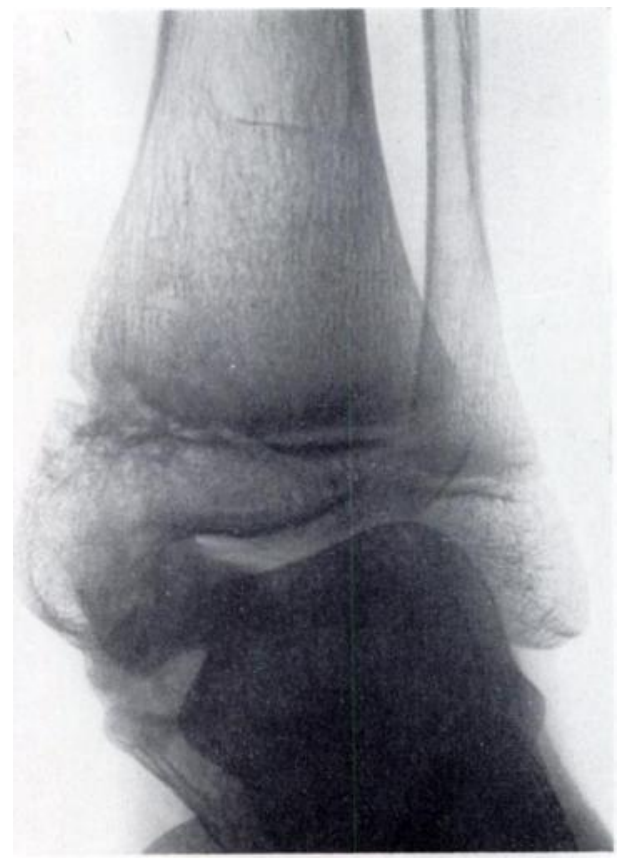

Fig. 7

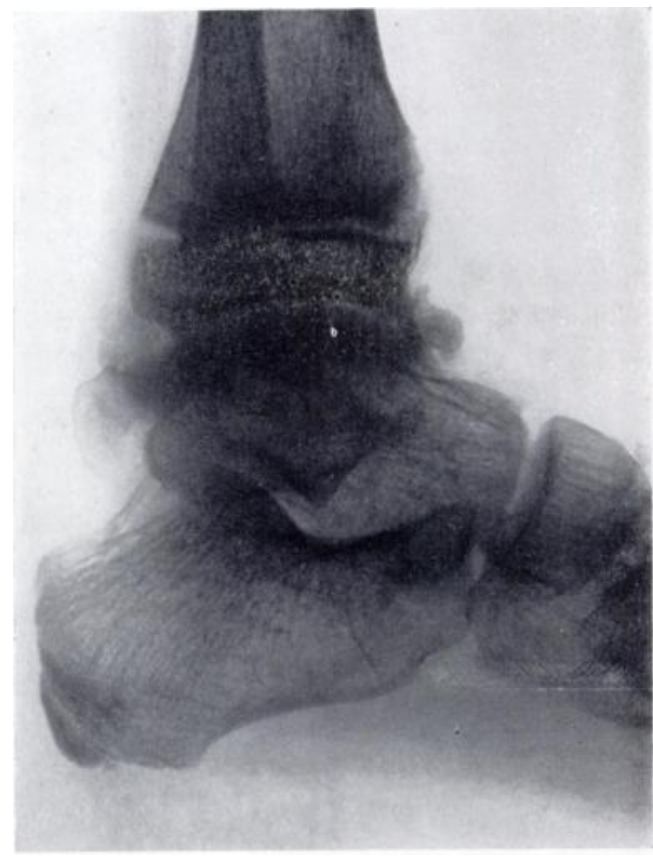

Fig. 8

Case 4. Figure 7-Fnlargement of the medial malleolus and medial half of the talus causing a valgus deformit. Figure 8 is a lateral view of the ankle showing that the mass is extending from the medial side of the talus anteriorly and posteriorly to the medial malleolus.

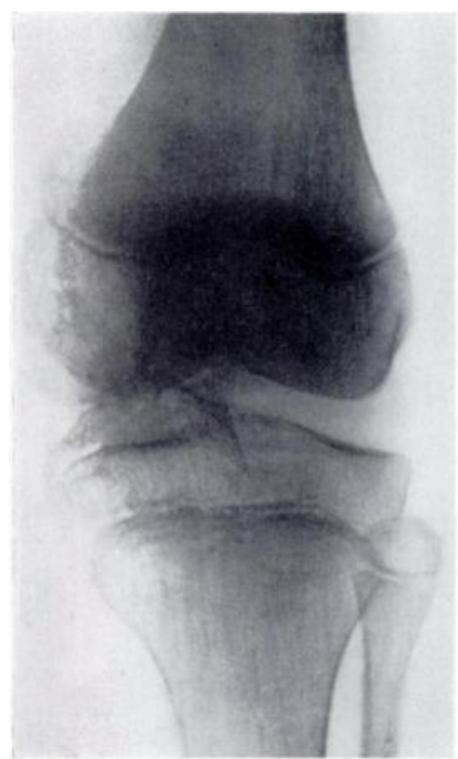

Fili. 9

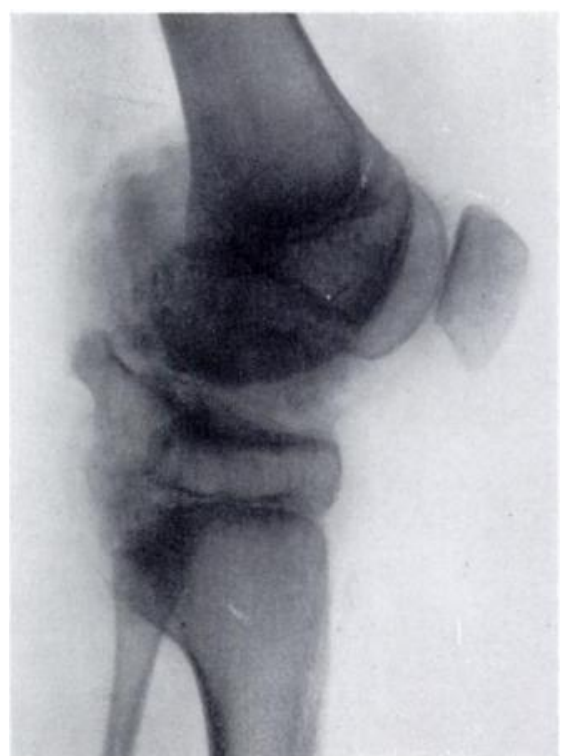

FIG. 10

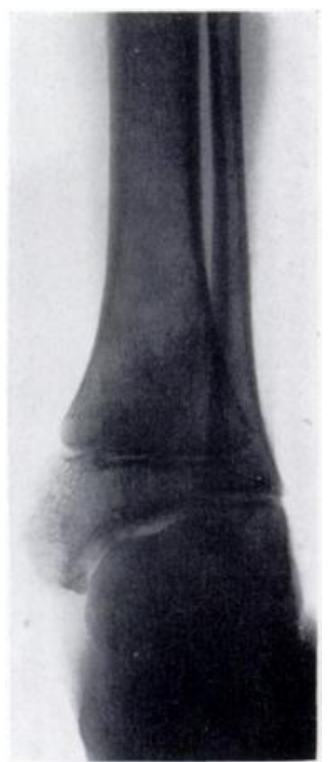

Fig. 11

Case 5. Figures 9 and 10 -.-Left knee showing enlargement of the medial femoral condyle and of the medial half of the upper tibial epiphysis. The swelling extends medially and backwards towards the popliteal space and has caused genu valgum. Figure 11 shows enlargement of the left medial malleolus and irregular bony outgrowth extending downwards towards the talus. This enlargement caused a valgus deformity. 
extension and flexion were limited; the total range of movement was approximately 60 degrees. Radiographs confirmed the clinical findings and showed also a small amount of backward subluxation of the tibia on the femur. Excision of the bony mass with arthrodesis of the knee was undertaken. Later examination showed an extreme valgus deformity of the left foot, and great restriction of ankle joint movements. Gross enlargement of the medial malleolus was found. At operation the talus was found to be involved extensively. The bony enlargements of the malleolus and talus were excised. Fifteen years later the patient was "looking well and walking well; he seems to have been earning his living for years." Case 5. (Under the care of the author. Figures 9, 10 and 11.) Boy aged seven years. Valgus deformity of the left foot and knee. On examination there was genu valgum with three inches separation of the malleoli. There was limitation of flexion of the knee joint, caused by a hard bony swelling which extended backwards from the medial side of the knee into the popliteal fossa. It was considered that this enlargement was the cause of the genu valgum. There was a similar but smaller swelling of the medial malleolus of the left ankle. There was a full range of movement of the ankle joint but inversion of the foot was restricted by the bony swelling. Radiographs confirmed the clinical findings. Operation was not advised, but valgus wedges were fitted to the left shoe. Three and a half years later the condition of the limb was unchanged. Disability was slight and the patient was leading a normal life.

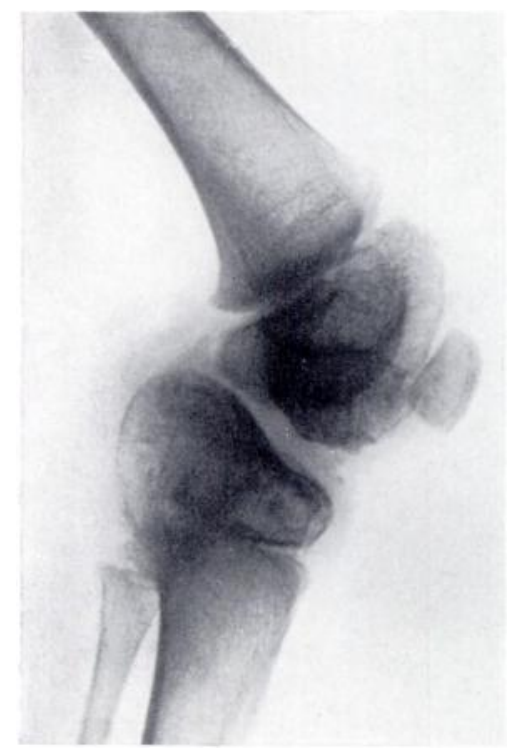

FIG. 12

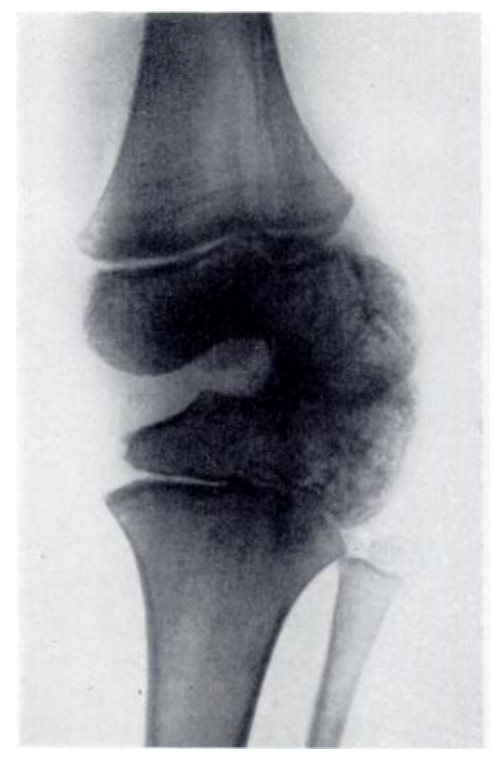

Case 6. Figure 12-Enlargement of the lateral condyle of the femur with enlargement of the corresponding half of the upper tibial epiphysis. The enlargement of the femoral condyle has occurred mainly in an anterior direction; that of the tibial epiphysis extends postero-laterally. The unilateral enlargements of the bone caused genu varum. Figure 13-Enlargement of the lateral malleolus and of the lateral part of the talus; the malleolar enlargement, which has several points of ossification, extends downwards and backwards.

Case 6. (Reported by the courtesy of Mr Sayle Creer. Figures 12 and 13.) Boy aged one and a half years when first seen. Parents noticed that he was walking badly, dragging his left leg and falling frequently. At that time a lateral curvature of the left tibia was noted. Six months later a swelling was noticed on the lateral side of the left knee. Radiographs showed a bony mass projecting from the lateral half of the lateral femoral condyle and from the tibial tuberosity.

The swelling was explored at operation. There was enlargement of the femoral condyle, which had many small nodules on its surface. The mass was not removed, but a small piece was taken for microscopic examination. Histologically it was composed of normal ossifying

VOL. $32 \mathrm{~B}$, NO. 2 , MAY 1950 
cartilage. A caliper was prescribed. When seen eight months later the lateral curvature of the left tibia had increased; movements of the knee joint were restricted to a range from 150-60 degrees. Two years later there had been no further increase of the deformity. The boy was leading an active life, complained only of an occasional ache in the knee, and extension movement had increased to within $\tilde{s}$ degrees of the full range. No further treatment was recommended. Later examination of his left ankle showed a similar enlargement of the lateral side. The swelling was symptomless and did not interfere with function.

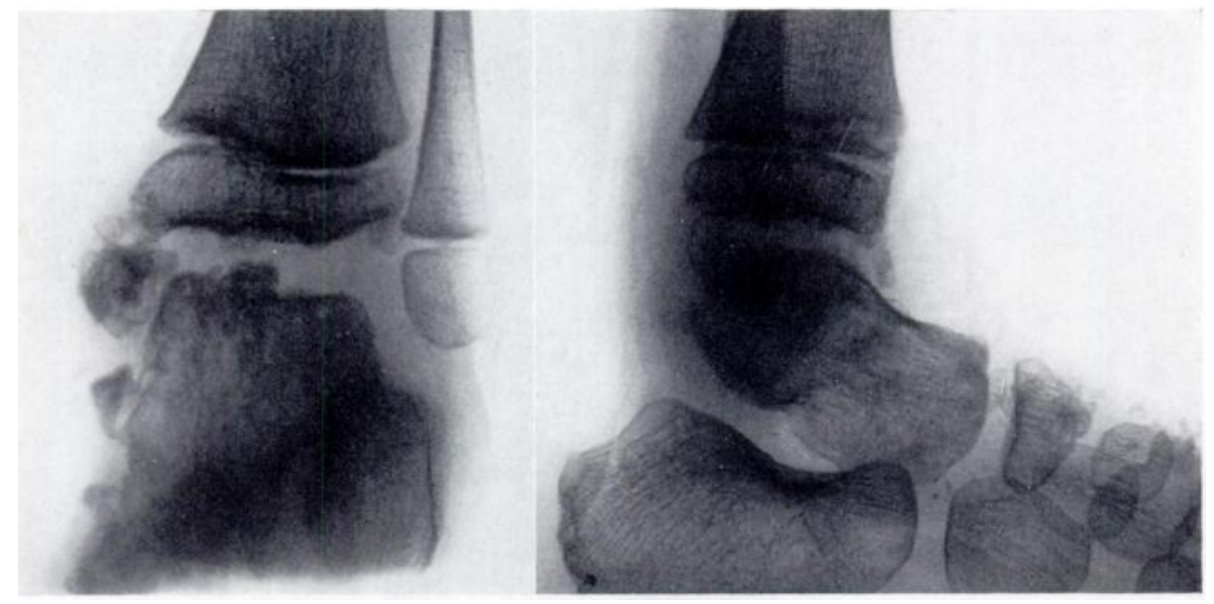

FIG. 14

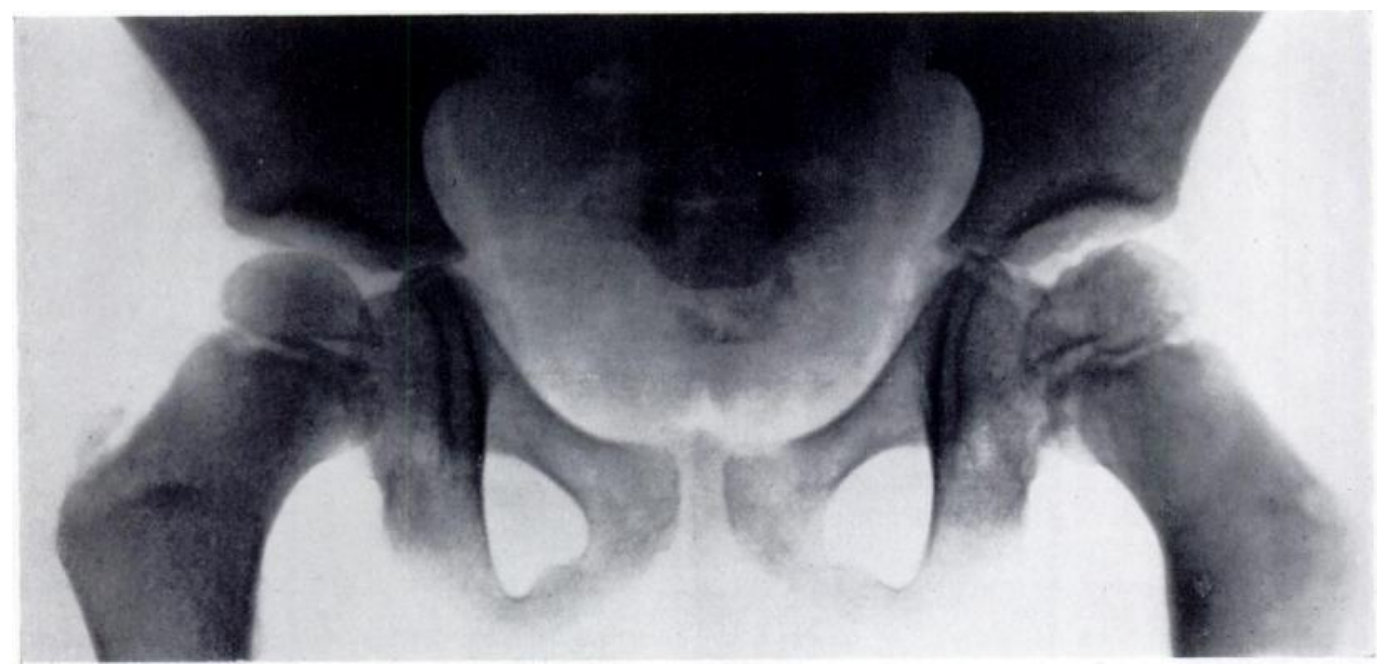

Fig. 15

Case 7. Figure 14-Enlargement of the medial malleolus and of the medial half of the talus, which is almost as large as the calcaneum. The navicular also is enlarged and there are several foci of ossification in the region of the medial cuneiform. Figure 15-Irregularity of the medial half of the left femoral head.

Case 7. (Reported by the courtesy of Mr Norman Capener. Figures 14 and 15.) Boy aged three years when first seen. Valgus deformity of the left foot, and no other symptoms. On examination there was enlargement of the medial malleolus of the left tibia. Radiographs showed enlargement of the medial half of the tibial epiphysis extending into the medial malleolus, which was increased in size and extended downwards towards the talus. The talus was enlarged, and an irregular bony outgrowth projected from its medial surface and from the medial half of its superior surface. The navicular and medial cuneiform 
presented similar appearances. Further radiographs showed enlargement of the medial femoral condyle and of the medial half of the femoral head. No other bones were affected.

When this boy was examined recently the condition of the left foot and ankle remained unchanged; there was a small enlargement of the medial femoral condyle but this did not interfere with the function of the knee joint and caused no deformity. The lower limbs were of equal length and girth. The boy was leading an active life, without disability. He was advised to wear valgus heel wedges but no other treatment was considered necessary.

Case 8. (Reported by the courtesy of Mr E. S. Evans. Figures 16 and 17.) Girl aged nine months when first seen. Mother had noticed that the child was unable to extend the right knee fully and that the right foot was deformed. On examination there was severe genu valgum and limitation of extension of the right knee. There was also gross valgus deformity of the right foot, but this could be corrected manually. Radiographs showed a bony outgrowth

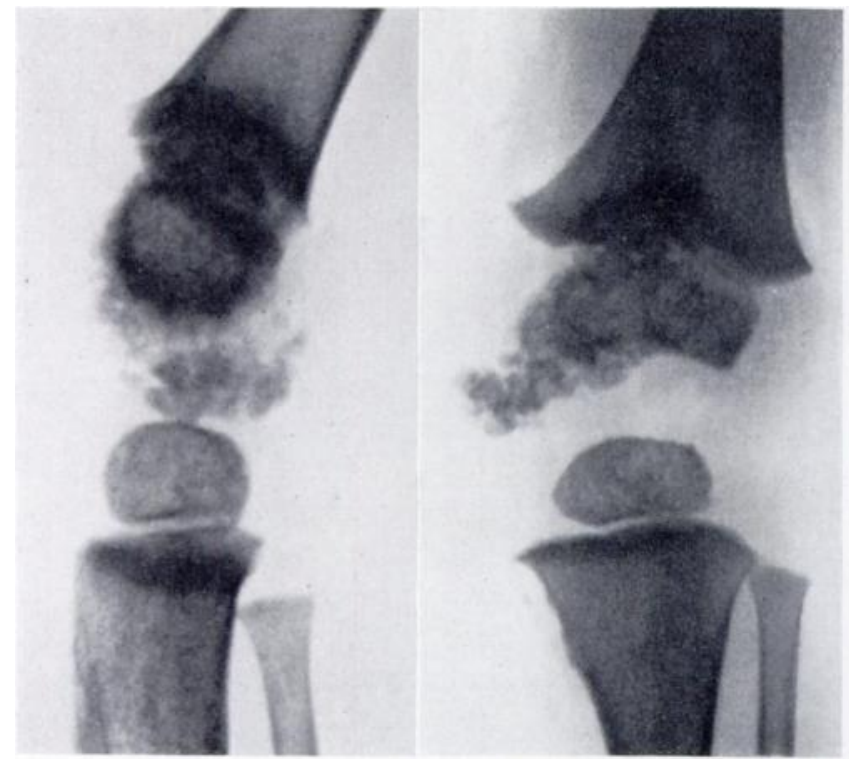

FIG. 16

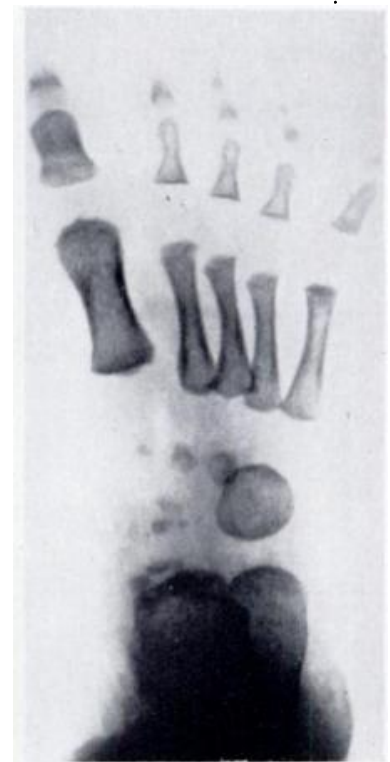

FIG. 17

Case 8. Figure 16-Enlargement of the medial femoral condyle. The cartilaginous-bony mass extends downwards, medially and backwards; there are several foci of ossification. Note the irregularity of the outline of the tibial shaft on the medial side in the region of the metaphysis. Figure 17-Right foot. Note the irregularity of the medial side of the talus and its enlargement, the multiple foci of ossification of the navicular and the early appearance of the ossific nuclei of the medial and middle cuneiforms.

from the medial femoral condyle with a mottled appearance due to multiple foci of ossification. The talus was enlarged and irregular outgrowths projected from its surface. The ossific centre for the navicular had appeared early and consisted of several separated foci; the ossific centres for the medial and middle cuneiform bones had also appeared prematurely.

Exploration of the medial side of the knee joint was undertaken at the age of two years. The medial femoral condyle was enlarged and smooth, but excision of the excess bone was not considered necessary. Supracondylar osteotomy was performed to correct the genu valgum. A lateral iron and medial $T$-strap were fitted to control the severe valgus deformity of the foot.

\section{DIAGNOSIS}

Tarso-epiphysial aclasis is clinically distinct from dyschondroplasia and from diaphysial aclasis, and no confusion should arise. Radiologically the distinction is obvious, for in dyschondroplasia and in diaphysial aclasis the essential changes are found in the metaphysis.

vol. $32 \mathrm{~B}$, No. 2 , MAY 1950 
Dysplasia epiphysialis punctata, although easily distinguished clinically, may be confused with tarso-epiphysial aclasis radiographically. In dysplasia epiphysialis punctata, particularly when the tarsus is involved, the stippling effect occurs in the epiphysis and extends throughout its whole width; the shafts of the long bones may be thickened and shortened and the metaphysis splayed. In tarso-epiphysial aclasis the stippling effect arises from the epiphysis and, in the case of the epiphyses of the long bones, from one half only; and the metaphysis appears normal.

\section{ETIOLOGY}

The condition is considered to be a congenital error of skeletal development. In the distribution of the lesions it resembles melorheostosis where one side of a limb is involved; in no case of this series was the medial side of the knee affected with the lateral side of the ankle and vice versa. It is suggested that the etiological factor affects the limb buds during early foetal life. This would explain the distribution: a pre-axial affection would produce the deformity on the medial side of the limb and a post-axial affection on the lateral side.

The arrangement of the blood vessels in the epiphyses (Fig. 18) may explain why the condition is confined to one half of the epiphysis. It may explain also the several foci of ossification that occur in the cartilaginous mass as it grows away from the epiphysis. According to Harris (1933) the cartilage of an epiphysis of a long bone is formed from a narrow zone where cartilage cells are actively dividing - a zone which he calls the mitotic annulus. At the centre of the cartilage mass, bony trabeculae of the ossific nucleus are produced. Surrounding this is a zone where degenerate cartilage cells are arranged in columns within a calcified matrix. And between this zone and the mitotic annulus there is a mass of cartilage cells, the youngest cells being adjacent to the mitotic annulus. The same arrangement occurs at the epiphysial line-successive zones extending from the mitotic annulus towards the shaft of a long bone consist first of young cells, older cells, and then degenerate cartilage cells arranged in columns with a calcified matrix, and lastly, diaphysial bone. But the arrangement of the cells between the mitotic annulus and the articular surface of the epiphysis is different. Adjacent to the mitotic annulus are the young, recently formed cartilage cells;

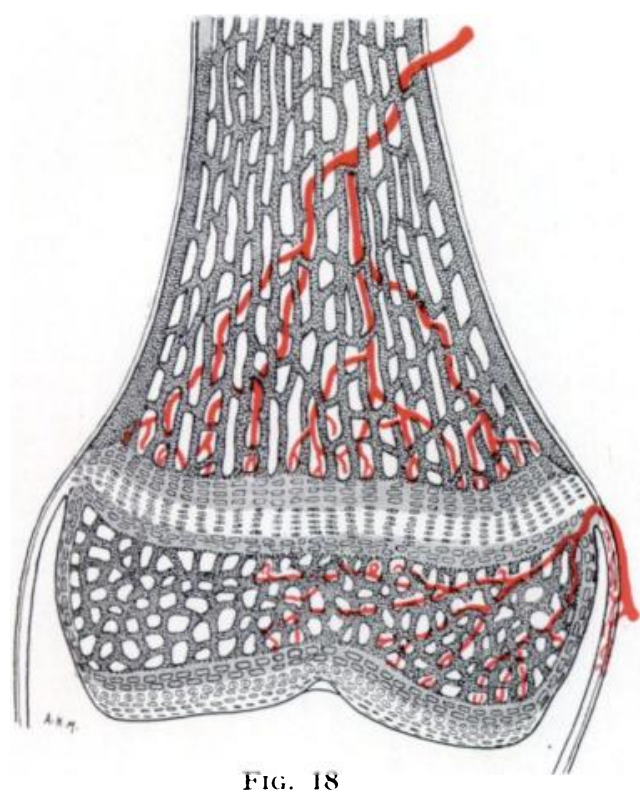

FIG. 18

Diagram showing arrangement of blood vessels supplying the epiphysis and metaphrsis in the lower end of the femur (from Harris). next are older cells more widely separated and flatter in outline: and at the articular surface the cells are completely flat and senescent. They eventually disintegrate and together with the matrix give rise to the main constituent of synovial fluid (Fig. 19). It is suggested that in tarso-epiphysial aclasis the process is altered and that the cells do not become senile and flat but retain their power of division, so that a mass of cartilage is produced. Later, calcification of the matrix occurs in discrete areas, which become ossified. Radiographically. these are seen as multiple foci of ossification.

It is probable that the pathology is the same in the case of the tarsus. The mitotic annulus produces cells on its central aspect which form the main cartilage mass of the bone, and the ossific nucleus forms within it. The cells produced peripherally normally become flattened and senile towards the surface, and finally disintegrate. Failure of these peripheral cells to degenerate in the normal way causes a mass of cartilage to grow from the 
peripheral surface and accounts for the generalised enlargement of a bone such as the talus.

It is because of the failure to confine these peripheral cartilaginous cells, derived from the mitotic annulus, that the term tarso-epiphysial aclasis is suggested for this affection.

\section{TREATMENT}

If the projecting mass can be removed without causing instability of the affected joint it should be removed; in Cases 1 and 2 the swelling was of a localised character and its removal produced a satisfactory end-result. But when the outgrowth is extensive its complete excision may necessitate stabilisation of the joint. This should be undertaken only if it is fully justified by the severity of the disability, and is seldom necessary. In Case 5 there was excellent function despite a large outgrowth on the postero-medial aspect of the knee and a smaller outgrowth on the medial side of the ankle. When a large bony outgrowth is associated with marked genu valgum it is advisable to remove as much as possible of the swelling without impairing the stability of the knee joint, and to correct the residual deformity by osteotomy. The treatment of outgrowths around the ankle joint is along similar lines, but when the enlargement and deformity are severe and affect the talus the parents should be warned that arthrodesis of the ankle joint may become necessary in later childhood.

I wish to thank all those surgeons who have readily given me permission to use the notes and radiographs of patients under their care. I am grateful in particular to Sir Thomas Fairbank for his constant helpfulness, kindly criticism and stimulation. It was only through his untiring efforts that it was possible to collect the cases in this series. Figures 18 and 19 are taken from H. A. Harris's "Bone Growth in Health and Disease "; my thanks are due to Professor Harris and to the publisher (Oxford University. Press).

\section{AUTHOR'S POSTSCRIPT}

Since this paper was submitted for publication, reports of two more patients afflicted with this condition have reached me through the kindness of Sir Thomas Fairbank. A six-year-old boy under the care of Mr R. H. Metcalfe had the inner side of the right ankle and foot involved; there was no functional disability, although he had to wear boots made to measure. The other patient is a four-year-old boy under the care of $\mathrm{Mr}$ Donald Brown of Newcastle-upon-Tyne; in this child, the medial left femoral condyle and the inner side of the right ankle and foot were affected. This is the only case of the series to show involvement of both lower limbs. Examination showed no other skeletal abnormality in either child. My thanks are due to Mr Metcalfe and to Mr Brown for permission to mention their cases.

\section{REFERENCES}

Harris, H. A. (1933): Bone Growth in Health and Disease. London: Humphrey Milford, Oxford University Press, 143.

Mouchet, A., and Belot, J. (1926): La tarsomégalie. Journal de Radiologie et d'Electrologie, 10, 289. 